\title{
Changes in Naming and Semantic Abilities With Aging From 50 to 90 years
}

Clémence Verhaegen AND Martine Poncelet

Department of Psychology: Cognition and Behavior, University of Liège, Liège, Belgium

(Received December 23, 2011; Final Revision July 23, 2012; Accepted July 27, 2012; First Published OnLine December 14, 2012)

\begin{abstract}
The aim of this study was to determine whether naming difficulties arise in individuals as young as their 50s. Participants of $25-35,50-59,60-69$, and above 70 years of age were given a picture naming task. To uncover subtle naming difficulties, latencies were analyzed in addition to accuracy. To control whether the expected slower naming latencies could be due to a general slowing affecting all cognitive tasks, participants were also given an odd/even judgment task to assess cognitive processing speed. The results confirmed that participants in their 50 s presented decline in naming performance, reflected by an increase in naming latencies, whereas adults in their $60 \mathrm{~s}$ and their 70 s showed both a decrease in accuracy and an increase in latency. Moreover, the increase in naming latencies remained significant even after controlling for odd/even judgment latencies, suggesting a degradation specific to the picture naming task. We assumed that these slower latencies may result from a language-specific impairment. As a further test for languagespecific degradation, participants' semantic capacities were also assessed with a synonym judgment task and the Pyramids and Palm Trees test. The above-70 group showed semantic degradation. The contributions of multiple factors to naming difficulties in aging are discussed. (JINS, 2013, 19, 119-126)
\end{abstract}

Keywords: Aging, Word naming difficulties, Picture naming, Semantic, Naming latencies, Age of onset

\section{INTRODUCTION}

One of the most frequent cognitive complaints among older people is difficulty finding words (Albert et al., 2009; Clark-Cotton, Williams, Goral, \& Obler, 2007; Hough, 2007; Mortensen, Meyer, \& Humphreys, 2006; Neumann, Obler, Gomes, \& Shafer, 2009). These difficulties have been confirmed in many studies showing a word naming decline on the Boston Naming Test (BNT), both cross-sectional (e.g., MacKay, Connor, Albert, \& Obler, 2002; Mariën, Mampaey, Vervaet, Saerens, \& De Deyn, 1998) and longitudinal (Au et al., 1995; Barresi, Nicholas, Connor, Obler, \& Albert, 2000; Connor, Spiro, Obler, \& Albert, 2004; Goral, Spiro, Albert, Obler, \& Connor, 2007).

Although older people complain of word-finding difficulties, not all experimental studies have found an age-related decline in naming. Moreover, the age at which the decrease in naming performance arises in the elderly is still a matter of debate. Indeed, after reviewing 25 picture naming studies, Goulet, Ska, and Kahn (1994) concluded that "age-related

Correspondence and reprint requests to: Clémence Verhaegen, University of Liège, Department of Psychology: Cognition and Behavior, B33 Boulevard du Rectorat, 4000 Liège, Belgium.

E-mail: clemence.verhaegen@ulg.ac.be decline in picture naming tasks is an inconsistent finding" (p. 629). They pointed out that the chances of finding a picture naming decline in aging may be influenced by many factors. For example, the age groupings used in different studies have varied considerably, the youngest participants ranging from 18 to 65 and the oldest from 59 to 85 years old. Moreover, they argued that, the results may have been influenced by the type of material used, such as the use of highly familiar items or a very low number of naming trials, probably resulting in relatively insensitive measures. Feyereisen (1997) presented contrasting findings in a metaanalysis of many of the studies reviewed by Goulet et al., selecting only studies that provided means and standard deviations for naming scores in 3 particular age groups -under 50, between 50 and 69 , and over 70 years old. Feyereisen concluded that a significant age-related decline in naming accuracy occurs only above the age of 70 . In contrast, Connor et al. (2004) and Nicholas, Connor, Obler, and Albert (1998) found subtle signs of decreased naming accuracy in participants in their 50s, although the naming decline appeared to become more pronounced in adults in their 70s.

It is also important to note that few studies have analyzed naming latencies in aged participants. For example, 
Morrison, Hirsh, and Duggan (2003), showed that older participants $(M=75.5$ years) responded more slowly on a picture naming task than younger ones ( $M=19.6$ years). Moreover, to our knowledge, no study has analyzed naming latencies in individuals in their 50s. We assume that such analyses are more likely to reveal a subtle naming decline in middle-aged participants than are analyses of naming accuracy.

The explanation of this age-related decline in naming also remains controversial. Two principal hypotheses have been proposed: the General Slowing theory and the Transmission Deficit Hypothesis (TDH).

According to General Slowing theories (e.g., Myerson, Ferraro, Hale, \& Lima, 1992; Salthouse, 1996), the elderly experience a general slowing of all cognitive processes, including language processes. According to Myerson et al. (1992), the mean response latencies of the elderly on speeded tasks are simply a multiplicative function of those of younger participants under the same conditions. A variant of this general slowing assumption is the view that age-related slowing varies across different task domains. For example, Lima, Hale, and Myerson (1991) reported a significantly greater degree of slowing in the non-lexical domain than in the lexical domain in older adults. Unfortunately, they did not offer an explanation of the difference between these two domains. Thus, according to the General Slowing theory, response time on cognitive tasks increases with age. However, the question of whether this slowing is constant across all cognitive tasks or whether it is different in each cognitive domain remains controversial.

The Transmission Deficit Hypothesis (Burke, MacKay, \& James, 2000; Burke, MacKay, Worthley, \& Wade, 1991; Burke \& Shafto, 2004; MacKay \& Burke, 1990) is more language-specific. It is based on an interactive activation model of language known as Node Structure Theory (NST). The NST postulates two hierarchically related systems of nodes: lexical-semantic and phonological. The nodes are interconnected inside each system and between these systems. The strength of the connections to a given node determines the rate and amount of priming transmitted to it and thus determines what information becomes available in production. According to the TDH, aging weakens the strength of the connections between nodes, resulting in a reduction of priming transmission and thus of word activation, ultimately leading to more word naming failures in the elderly.

According to Goral et al. (2007), the TDH can account for picture naming difficulties in the elderly. Nicholas, Obler, Albert, and Goodglass (1985) conducted a naming study using the BNT in participants between 30 and 79 years old. In case of naming failure, participants were presented a phonemic cue. They found that the number of occasions where phonological cues were needed increased with age. Nevertheless, the benefit of cueing was the same at all ages. Nicholas et al. argued that this equal benefit from phonological cueing showed that the older participants' phonological representations were intact, but their access to phonological information was disrupted, leading to more naming difficulties. With the aid of phonological cues they could overcome this disruption. However, in a longitudinal study using the same paradigm, Au et al. (1995) found that adults above 70 years of age benefited less from phonemic cueing than younger participants. Au et al. hypothesized that the decline in naming ability across the lifespan may reflect not only a connectional deficit between lexical-semantic and phonological systems, but a subtle lexical-semantic degradation in the elderly.

Attempting to test the hypothesis of Au et al., Barresi et al. (2000) tried to discriminate between semantic degradation (i.e., a semantic system in which concepts are weakly represented or weakly interconnected) and impaired access to phonological forms by investigating the consistency of naming failures on the BNT in a longitudinal study with participants in their 50s, 60s, or 70s. Participants performed three sessions at 3- to 3.5-year intervals. The authors considered that successful naming or naming after cueing in an early test session followed by failure to name an item after a phonological cue in a later test session may indicate semantic degradation, whereas they interpreted failure in an early test session followed by successful naming or cueing in a later test session as an indication of impaired access to the phonological form. The results showed that the younger two age groups (50s and 60s) produced mainly naming failures of the type attributed to impaired phonological access, whereas both types of naming failures were observed in the participants above 70 years of age. The authors concluded that age-related naming failures may be due not only to impaired phonological access, but also in part to semantic degradation. Finally, according to Barresi et al. (2000), these results are compatible with the TDH, according to which "aging weakens connections within the entire network of nodes, reducing the rate and amount of priming transmitted across connections" (Burke et al., 1991, p. 545).

In sum, the age-related decline in naming remains a controversial subject. First, the age at which naming difficulties arise is still debated. Indeed, according to Feyereisen (1997), the significant decline in naming occurs after the age of 70 . However, Connor et al. (2004) and Nicholas et al. (1998) found subtle naming difficulties in people in their 50s. Second, the explanation for these naming failures in aging is also debated. According to some authors (e.g., Salthouse, 1996), these failures result from a general slowing on all cognitive tasks in the elderly, including language; others argue instead for a more language-specific explanation, attributing the decline in naming performance to a weakening of connections across the entire language system, including inside the semantic system (Barresi et al., 2000; Burke et al., 1991).

The first aim was to determine whether there is in fact an age-related decline in naming in the elderly, and particularly whether these difficulties arise in individuals in their 50s. Participants of 25-35, 50-59, 60-69, and above 70 years of age were given a picture naming task. To check for subtle naming difficulties, both naming accuracy and naming latencies were analyzed. We predicted that the naming difficulties of participants in their 50s in particular might be subtle and could be revealed by longer naming latencies 
Table 1. Demographic data of the $25-35,50-59,60-69$ and above-70 age groups

\begin{tabular}{|c|c|c|c|c|c|}
\hline Variable & Age group & $25-35$ years & $50-59$ years & $60-69$ years & Above 70 years \\
\hline No. of participants & & 30 & 30 & 30 & 30 \\
\hline Age (years) ${ }^{\mathrm{a}}$ & & $28.87(2.94)$ & $54.40(3.42)$ & $63.77(2.60)$ & $80.67(5.25)$ \\
\hline Gender (M/F) & & $14 / 16$ & $8 / 22$ & $14 / 16$ & $14 / 16$ \\
\hline \multirow[t]{3}{*}{ Socio-economic background ${ }^{\mathrm{b}}$} & Level $1^{\mathrm{c}}$ & 0.27 & 0.23 & 0.27 & 0.27 \\
\hline & Level $2^{c}$ & 0.23 & 0.30 & 0.27 & 0.30 \\
\hline & Level $3^{c}$ & 0.50 & 0.47 & 0.47 & 0.43 \\
\hline Mattis Scale $(/ 144)^{\mathrm{a}}$ & & - & $143.5(0.94)$ & $143.07(1.23)$ & $140.10(2.77)$ \\
\hline Mill Hill $(/ 33)^{\mathrm{a}}$ & & $26.10(2.55)$ & $26.30(3.60)$ & $27.73(3.99)$ & $25.60(4.73)$ \\
\hline
\end{tabular}

${ }^{\mathrm{a}}$ Means and standard deviations in parentheses.

${ }^{\mathrm{b}}$ Proportions.

${ }^{\mathrm{c}} 1=$ weak, $2=$ middle and $3=$ high

unaccompanied by any decrease in naming accuracy. We assumed that the age-related naming disadvantage may increase with age (Connor et al., 2004), with the eventual onset of naming errors. Thus, in participants above 70 years old, we expected to find both slower naming latencies and lower picture naming scores. We assumed that the naming difficulties in participants in their 50s, their 60s, and above 70 years old may result from a language-specific impairment (TDH, Burke et al., 1991). However, because we analyzed naming latencies, we wanted to control whether these expected slower latencies may result from a general slowing that affects all cognitive tasks equally (Salthouse, 1996). For that purpose, participants were also given an odd/even judgment task, to assess cognitive processing speed.

The second aim was to seek further support for the hypothesis of a language-specific impairment in aging by assessing participants' semantic capacities. Indeed, Barresi et al. (2000) reported semantic degradation in people in their 70s. In our study, we used more direct methods to evaluate the semantic system: the Pyramids and Palm Trees Test (Howard \& Patterson, 1992) and a synonym judgment task. We expected to find lower semantic scores in participants in their 70s.

\section{METHOD}

\section{Research Participants}

Four groups of participants took part in the present study: (1) 30 participants between 25 and 35 years of age $(M=28.87 ; S D=2.94)$, (2) 30 between 50 and 59 years of age $(M=54.40 ; S D=3.42)$, (3) 30 between 60 and 69 years of age $(M=63.77 ; S D=2.59)$, and (4) 30 above 70 years of age $(M=80.67 ; S D=5.25)$. Participants responded to a questionnaire on their health and reported no history of neurological, cardiac, neuropsychological, or psychiatric disorders, and no uncorrected hearing or visual problems. They were also carefully screened for medication use. Participants taking antidepressants or other psychoactive medications were excluded from the study. Participants above 50 years of age were given the Mattis Dementia Rating Scale
(Schmidt et al., 1994) and performed above the cutoff score of 130/144. All participants were native speakers of French. There were no reliable differences between the 4 groups in the distribution of socio-economic backgrounds. Participants were classified into 3 socio-economic levels: (1) low, (2) middle, and (3) high, according to the classification of Amos et al. (2003) which determines socio-economic level on the basis of participants' years of schooling and profession. A chi-squared test showed no significant relationship between socio-economic background and group, $\chi^{2}(6, n=120)=.57, n s$. Participants' level of vocabulary was evaluated using the Mill Hill test (Deltour, 1993). An analysis of variance (ANOVA) computed on the number of correct responses out of 33 revealed that all age groups performed equally, $F(3,116)=1.74$, ns. The local research ethics committee approved the study, and all human data included in this manuscript was obtained in compliance with the Helsinki Declaration. All participants gave their informed consent. Demographic data are summarized in Table 1.

\section{Materials}

\section{Picture naming task}

One hundred fifty black and white line drawings were selected from the set of Bonin, Peereman, Maladier, Méot, and Chalard (2003). Bonin et al. reported a name agreement percentage for each picture, consisting of the percentage of participants who produced the most common name. On the basis of these results, we selected pictures with a name agreement higher than $60 \%$. In the present study, each picture was presented centered on the computer screen using the E-Prime 2.0 software package (Psychology Software Tools). Participants were asked to name each picture as quickly as possible. The pictures were presented in a pseudo-randomized order. A ready signal ("*") appeared at the center of the screen for $500 \mathrm{~ms}$ and was followed by a 100-ms tone, which ended at the onset of the picture. The experimenter then pressed a button to begin the next trial. Participants were given a short break after every 45 trials. Fifteen additional pictures were used as warm-ups. Standard phonemic and 
semantic cues were provided in case of naming failure. ${ }^{1}$ The test session was recorded and transcribed for scoring. The accuracy score consisted of the number of correctly named items $^{2}$ (of a total of 150). An item was counted as correct if the participant named the item correctly and spontaneously. Self-corrected naming errors were accepted. Correct responses after a cue were not included in the number of correct responses. The warm-up trials were not included in the score. Cueing effect differences were not analyzed given the small number of naming failures in the 3 younger groups. Correct naming latencies (in ms) were analyzed with the Audacity 1.2.6. software (Mazzoni, 2006). Correct naming latencies consisted of the latencies between the presentation of the picture and the items named correctly and spontaneously, without any cue.

\section{Cognitive processing speed task: the odd/even judgment task}

In this task from Schyns and Poncelet (2002), 50 digits between 1 and 9 were presented in random order, centered on the computer screen, using the E-Prime 2.0 software. Participants had to indicate whether the digits were odd or even by pressing a designated "odd" or "even" key as quickly and accurately as possible. Ten additional digits were used as warm-ups. Response latencies were recorded by the computer.

\section{Semantic assessment}

The participants' semantic capacities were assessed using two tasks chosen to tap different aspects of semantic knowledge through different modalities: the Pyramids and Palm Trees test (PPTT; Howard \& Patterson, 1992), which requires participants to match concrete pictures, and a synonym judgment task (adapted from Majerus, Lekeu, Van der Linden, \& Salmon, 2001), which requires the semantic processing of abstract vs. concrete auditory words.

The Pyramids and Palm Trees test. Participants were presented 52 plates of three object-pictures each, one above the other two. They were asked to indicate which of the bottom two pictures had the closest semantic relationship with the target. Three additional plates were used as warmups and were not included in the scoring. The number of correct responses (out of 52) was computed.

Synonym judgment task. Sixty pairs of concrete and abstract words were presented orally by means of headphones; participants had to decide whether the words had similar meanings and were asked to respond by pressing a

\footnotetext{
${ }^{1}$ The cues were administered automatically. If participants clearly misperceived the object, they were provided with a semantic cue. If the response was semantically related but incorrect, they were asked to find "another word" for the target picture. If the participants gave an incorrect response or were unable to give a response within the cutoff response time of 10 seconds, they were provided with a phonological cue (the first phoneme of the word).

${ }^{2}$ Because name agreement on some of the pictures was as low as $60 \%$, we accepted alternative names for the pictures with more than one acceptable name, in keeping with Bonin et al.'s (2003) list.
}

designated key as quickly and accurately as possible. The pairs were matched for imageability (Desrochers \& Bergeron, 2000). Three additional trials were used as warm-ups and were not included in the scoring. This task was programmed and presented with the E-Prime 2.0 software. The number of correct responses (out of 60) was recorded by the computer.

\section{Procedure}

The whole study was conducted in French. Participants were tested individually. The order of the tasks was constant across participants: (1) Picture naming task, (2) Synonym judgment task, (3) PPTT, (4) Odd/even judgment task, (5) Mill Hill, (6) Mattis Dementia Rating Scale. The experiment took approximately $90 \mathrm{~min}$ to complete and was performed in a single session.

\section{RESULTS}

\section{Age-Related Effects on Naming Accuracy}

Table 2 shows mean performance (number of correct responses) on the picture naming task for the 25-35, 50-59, 60-69 and above-70 age groups. An ANOVA performed on the number of correctly named items (out of 150 ) revealed an effect of age, $F(3,116)=35.36, p<.001 ; \eta_{\mathrm{p}}^{2}=.48$. NewmanKeuls post hoc comparisons $(p<.05)$ indicated that the above70 age group named fewer items correctly than the 60-69 age group, which performed worse than the 25-35 and 50-59 age groups, which in turn did not differ from each other. In sum, these results indicate a naming decrease in the 60-69 and above70 age groups.

\section{Age-Related Effects on Response Latencies on the Picture Naming and Odd/Even Judgment Tasks}

Figure 1 shows mean response latencies on the picture naming and odd/even judgment tasks for the 25-35, 50-59, $60-69$, and above-70 age groups. For the picture naming task, correct naming latencies were analyzed. For the odd/ even judgment task, analyses were performed on all response latencies because the error rate was very low (1.35\%). Outlier latencies (more than 2 standard deviations from the mean of the latencies for the same subject) were also removed from the analyses for both tasks. We performed a mixed ANOVA with a 4 (Age group: 25-35, 50-59, 60-69, and above70) $\times 2$ (Task: picture naming task, odd/even judgment task) design on log-transformed response latencies in order both to examine the main effects of age group and task and also to investigate whether there was an interaction between these two variables. There was a significant effect of age, $F(3,116)=129.9, p<.001 ; \eta_{\mathrm{p}}^{2}=.77$. Newman-Keuls post hoc comparisons $(p<.05)$ revealed that on the odd/even judgment task, the $25-35$ and 50-59 age groups did not differ from each other, both responding faster than the 60-69 and above-70 age groups, which also did not differ from each other. 
Table 2. Mean performance (number of correct responses out of 150) on the picture naming task in the 25-35, 50-59, 60-69, and above-70 age groups

\begin{tabular}{|c|c|c|c|c|c|c|c|c|}
\hline & \multicolumn{2}{|c|}{ 25-35 years } & \multicolumn{2}{|c|}{$50-59$ years } & \multicolumn{2}{|c|}{$60-69$ years } & \multicolumn{2}{|c|}{ Above 70 years } \\
\hline & $M$ & $S D$ & $M$ & $S D$ & $M$ & $S D$ & $M$ & $S D$ \\
\hline Picture naming & 147.80 & 2.19 & 143.97 & 6.89 & 135.50 & 6.30 & 114.60 & 25.58 \\
\hline
\end{tabular}

In contrast, on the picture naming task, the $25-35$ age group responded faster than the 50-59 and 60-69 age groups, which did not differ from each other. The above-70 age group responded more slowly than the 3 younger groups. The effect of task was significant, $F(1,116)=1199.7, p<.001 ; \eta_{\mathrm{p}}^{2}=.91$, with participants responding faster on the odd/even judgment task than on the picture naming task. Finally, the age by task interaction was significant, $F(3,116)=39.40, \quad p<.001$; $\eta_{\mathrm{p}}^{2}=.50$. Planned comparisons with Bonferroni corrections for multiple comparisons (0.025) revealed that all groups responded more slowly in the picture naming task than in the odd/even judgment task, all $p s<.001$. However, this slowing was greater in the above-70 age group than in the 25-35, 50-59, and 60-69 age groups. In sum, difference on the picture naming task appears to be greater and to arise earlier in the lifespan than slowing on the odd/even judgment task.

An analysis of covariance was also performed on logtransformed naming latencies, using log-transformed response latencies on the odd/even judgment task as covariate. There was a significant effect of age, $F(4,115)=54.56$, $p<.001 ; \eta_{\mathrm{p}}^{2}=.46$. Newman-Keuls post hoc analysis indicated that the 25-35 age group responded faster than the 50-59 and 60-69 age groups, which did not differ from each other. The above-70 age group performed more slowly than the 3 younger groups. Thus, participants above 50 years of age showed a slowing of picture naming latencies. This slowing remains significant even after controlling for cognitive processing speed assessed by the odd/even judgment task.

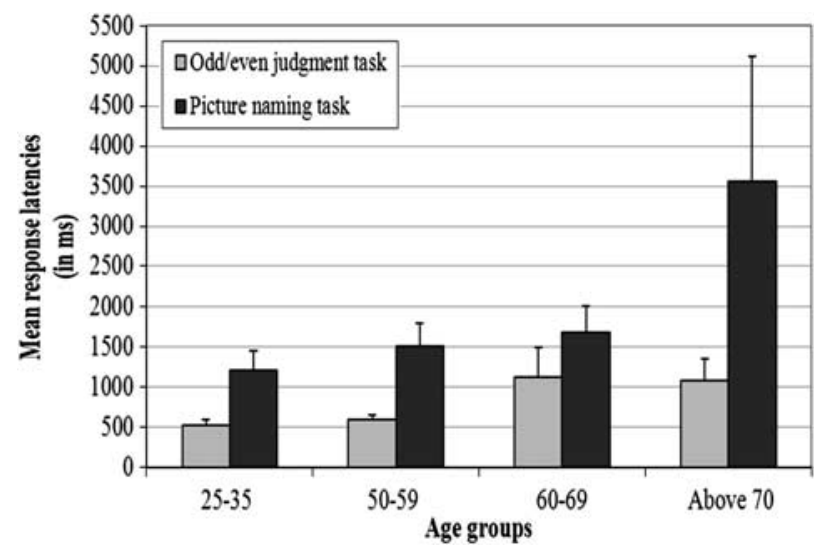

Fig. 1. Mean response latencies on the picture naming and odd/even judgment tasks in the 25-35, 50-59, 60-69, and above-70 age groups.

\section{Effects of Aging on Semantic Capacities}

Table 3 gives the mean number of correct responses on the PPTT (out of 52) and the synonym judgment task (out of 60) for the $25-35,50-59,60-69$, and above-70 age groups. Two ANOVAs were performed on the number of correct responses on each task. There were significant effects of age on the PPTT, $F(3,116)=20.43, p<.001 ; \eta_{\mathrm{p}}^{2}=.35$, and the synonym judgment task, $F(3,116)=22.78, p<.001 ; \eta_{\mathrm{p}}^{2}=.37$. Newman-Keuls post hoc comparisons $(p<.05)$ indicated that the above-70 age group performed worse than the 3 younger groups, which did not differ from each other on either of the semantic tasks. Thus, semantic performance appeared to be impaired in the above-70 age group.

\section{Correlation Analyses}

The results reported above indicate a degradation of both naming and semantic capacities in the above-70 age group. The relationship between the two types of degradation was examined using correlation analyses on naming and semantic capacities in the above-70 age group: between the number of correct responses on the picture naming task and the number of correct responses on the PPTT and the synonym judgment task, as well as between the naming latencies on the picture naming task and the number of correct responses on the two semantic tasks. Correlation analyses were not performed for the younger groups due to ceiling effects. As indicated in Table 4, all Bravais-Pearson correlations were significant in the above-70 age group.

\section{DISCUSSION}

\section{Effects of Aging on Naming Capacities: Age of Onset and Theories of Naming Decline in Aging}

The first aim of this study was to confirm the presence of naming difficulties in aging and to investigate whether the onset of these difficulties occurs in adults in their 50s. To this end, participants of 25-35, 50-59, 60-69, and above 70 years of age were given a picture naming task.

First, we analyzed the effect of age on naming accuracy. Participants in their 60s gave fewer correct responses than the 25-35 and 50-59 age groups, and the decrease was greater in individuals in their $70 \mathrm{~s}$.

The naming scores of participants in their 50s were not lower than those of the 25-35 age group. To check for more subtle naming difficulties in these participants, we analyzed 
Table 3. Mean performance (number of correct responses) on the Pyramids and Palm Trees Test (PPTT) and the synonym judgment task in the $25-35,50-59,60-69$, and above-70 age groups

\begin{tabular}{|c|c|c|c|c|c|c|c|c|}
\hline & \multicolumn{2}{|c|}{$25-35$ years } & \multicolumn{2}{|c|}{$50-59$ years } & \multicolumn{2}{|c|}{$60-69$ years } & \multicolumn{2}{|c|}{ Above 70 years } \\
\hline & $M$ & $S D$ & $M$ & $S D$ & $M$ & $S D$ & $M$ & $S D$ \\
\hline PPTT (/52) & 50.37 & 1.30 & 50.50 & 1.59 & 50.60 & 1.35 & 46.77 & 3.79 \\
\hline Synonym judgment (/60) & 57.73 & 1.81 & 58.03 & 1.35 & 57.83 & 1.34 & 54.57 & 2.74 \\
\hline
\end{tabular}

correct naming latencies. Moreover, we wanted to control whether such slower naming latencies may be explained by a general slowing in all cognitive domains (Salthouse, 1996). Participants were also given an odd/even judgment task, assessing cognitive processing speed. The results showed an effect of age on both tasks. On the picture naming task, adults in both their 50s and 60s were slower than the 25-35 age group, a slowing that became much greater in the above- 70 age group. By contrast, on the odd/even judgment task, participants in their 50s did not differ in speed from the 25-35 age group, and they responded faster than the two older groups. Moreover, all participants performed more slowly on the picture naming task than on the odd/even judgment task and this task effect was greater for the above70 age group than for the younger groups.

Although participants in their 50s made no more errors than younger participants, their significantly greater correct naming latencies suggest a subtle decline in naming at this age. In contrast, in participants in their 60 s and 70 s, the occurrence of an age-related decline in naming performance was confirmed by both a decrease in the mean number of correct responses and an increase in correct naming latencies. These findings are consistent with the results of the many studies that have found naming difficulties in aging (e.g., Goral et al., 2007; Mariën et al., 1998). However, the naming difficulties seem to arise earlier than suggested by Feyereisen (1997) who concluded that these difficulties appear after the age of 70. Our findings seem to be more consistent with the findings of Connor et al. (2004) and Nicholas et al. (1998), who reported subtle age-related naming difficulties in participants in their 50s. Our results highlight the importance of analyzing picture naming latencies, and also of comparing groups of 50-59, 60-69, and above 70 years of age, as some changes in naming abilities may occur between these decades.

Table 4. Bravais-Pearson correlations between the picture naming task, the Pyramids and Palm Trees test (PPTT), and the synonym judgment task in the above-70 age group

\begin{tabular}{lccc}
\hline \hline & \multicolumn{1}{c}{ PPTT } & Synonym judgment \\
\cline { 2 - 3 } & Correct responses & Correct responses \\
\hline Picture naming task & & $.49^{*}$ \\
Correct responses & $.40^{* *}$ & $-.56^{* *}$ \\
Correct naming latencies & $-.60^{* *}$ & \\
\hline \hline
\end{tabular}

$* p<.01 ; * * p<.001$.
The results of the mixed ANOVA suggest that slowing begins earlier in the lifespan on the picture naming task (in participants in their 50s) than on the odd/even judgment task (in participants in their 60s). Furthermore, the slowing in picture naming latencies increased importantly in participants above 70 years of age, as shown by the interaction. Finally and importantly, the slowing of correct naming latencies beginning in participants in their 50s remained significant even when processing speed assessed by the odd/even judgment task was controlled for using an ANCOVA. Thus, the slower naming latencies in participants in their 50s cannot be attributed to a general, task-independent slowing.

The results of the ANCOVA suggest that the slowing on the picture naming task may be explained by an impairment that is specific to this particular task, of linguistic origin. This impairment may reflect slower activation of language representations due to a weakening of connections throughout the language system (Burke et al., 1991). In participants in their 50 s, this weakness may remain subtle; these participants may be able to activate the word, but do so more slowly than before. As Connor et al. (2004) pointed out, the age-related decline in naming may accelerate with increasing age. In participants in their 60s, connections may have further weakened, leading to a decrease in the mean number of correct responses and slower correct naming latencies. Finally, in the above-70 age group, the decline may have become more pronounced, resulting in more naming errors and longer correct naming latencies.

However, the slowing in the odd/even judgment task became significant in participants in their 60s and above 70 years of age, which leads us to assume that both general slowing and language-specific factors may explain their naming difficulties.

Moreover, other non-linguistic factors may have contributed to the specific slowing on the picture naming task, such as a slowing of the perceptual analysis due to the age-related perceptual analysis difficulties.

In conclusion, several interacting mechanisms may contribute to word naming difficulties in aging.

\section{Age-Related Effect on Semantic Capacities}

The second aim of this study was to confirm the presence of semantic degradation in the elderly, as highlighted by Au et al. (1995) and Barresi et al. (2000). Au et al. (1995) hypothesized that the decrease in the phonemic cueing effect found in adults above 70 years of age is due to lexical-semantic 
degradation in the elderly. This hypothesis was confirmed by Barresi et al. (2000), who examined the consistency of naming failures across each of a series of three test sessions undergone by participants in their 50s, 60s, and their 70s. Barresi et al. interpreted consistent naming failures as indicative of semantic degradation and inconsistent naming failures as suggesting impaired phonological access. Their findings led them to conclude that naming failures in the elderly may be attributed both to impaired phonological access and to semantic degradation, and that they are compatible with the TDH (Burke et al., 1991), assuming weakened connections throughout the entire language system. However, Barresi et al. did not directly assess semantic capacities. In our study, participants performed semantic tasks that we assumed would tap directly into the semantic system: the PPTT and a synonym judgment task. The first is a picture matching task with concrete words, whereas the second consists of judgments of synonymy for auditory concrete and abstract words. We could not analyze the integrity of phonological access using differences in phonological cueing effects between age groups, however, because of the small number of naming failures in the three younger groups. The results confirmed that participants above 70 years of age made more errors than the three younger groups on both semantic tasks.

According to the TDH, to produce a word, semantic knowledge about it is required (Clark-Cotton et al., 2007). This hypothesis suggests specific relationships between word production and semantic capacities. Participants above 70 years of age presented impaired results on both tasks. To test these relationships, we performed correlation analyses between the number of correct responses on the picture naming task and the number of correct responses on both the PPTT and synonym judgment tasks, as well as between naming latencies on the picture naming task and number of correct responses on both semantic tasks in participants above 70 years of age. All correlations were significant, and together they suggest a relationship between naming and semantic capacities in the elderly. However, the nature of this relationship cannot currently be specified. Future studies could seek to do so by using the same words on both the semantic and picture naming tasks. A selective impairment for particular words on both tasks would suggest a possible relationship between semantic representations and production difficulties for these words (Hillis, Rapp, Romani, \& Caramazza, 1990; Samson, Pillon, \& De Wilde, 1998). However, it is also possible that an age-related general degradation contributed to these correlations.

As discussed above, the age-related naming difficulties of individuals in their 50s and beyond may be explained by a weakening of connections in the language system. With regard to semantic capacities, we hypothesized that this weakening may not have proceeded far enough in participants in their 50s and their 60s to create lower semantic scores. By contrast, in participants above 70 years of age, the connection deficit may become greater and more widespread, resulting in lower semantic scores.

\section{CONCLUSION}

In conclusion, the increase in correct naming latencies on the picture naming task in participants in their 50s suggests the presence of a subtle age-related decline in word naming. In participants in their 60s, naming degradation was highlighted by both a decrease in correct responses and an increase in naming latencies. Finally, in participants above 70 years of age, this decline became more pronounced in both naming accuracy and naming latencies. Moreover, only participants above 70 years of age presented semantic impairment. These results seem to suggest the presence of an age-related decline at the semantic level in the language system of older participants, and are compatible with the findings of Barresi et al. (2000). The slowing on the picture naming task seen in participants in their 50s remained significant after controlling for speed of responding on the odd/ even judgment task, suggesting a slowing specific to the picture naming task. These results may be explained by language-specific changes or by other non-linguistic factors. Future studies should explore the underlying cognitive processes that explain this decrease in normal aging while comparing participants in their 50s, 60s, and in their 70s.

\section{ACKNOWLEDGMENTS}

Support for this research was provided by the University of Liège (doctoral fellowship for fields not eligible for FRIA). We thank Vinciane Kaivers for her contribution in data collection. There are no conflicts of interest affecting this manuscript.

\section{REFERENCES}

Albert, M.L., Spiro, A., Sayers, K.J., Cohen, J.A., Brady, C.B., Goral, M., \& Obler, L.K. (2009). Effects of health status on word finding in aging. Journal of the American Geriatrics Society, 57, 2300-2305.

Amos, J., Jaunin, A., Le Roy-Zen Ruffinen, O., Lurin, J., Petrucci, F., \& Pillet, M. (2003). Relever les défis de la société de l'information-Rapport Genevois ALL. Genève, Switzerland: Service de la recherche en éducation.

Au, R., Joung, P., Nicholas, M., Obler, L.K., Kass, R., \& Albert, M.L. (1995). Naming ability across the adult life span. Aging and Cognition, 2(1), 300-311.

Barresi, B., Nicholas, M., Connor, L.T., Obler, L.K., \& Albert, M.L. (2000). Semantic degradation and lexical access in age-related naming failures. Aging, Neuropsychology, and Cognition, 7(3), 169-178.

Bonin, P., Peereman, R., Maladier, N., Méot, A., \& Chalard, M. (2003). A new set of 299 pictures for psycholinguistic studies: French norms for name agreement, image agreement, conceptual familiarity, visual complexity, image variability, age of acquisition, and naming latencies. Behavior Research Methods, Instruments, \& Computers, 35(1), 158-167.

Burke, D.M., MacKay, D.G., \& James, L.E. (2000). Theoretical approaches to language and aging. In T. Perfect \& E. Maylor (Eds.), Models of cognitive aging (pp. 204-237). Oxford, UK: Oxford University Press.

Burke, D.M., MacKay, D.G., Worthley, J.S., \& Wade, E. (1991). On the tip of the tongue: What causes word finding failures in young and older adults? Journal of Memory and Language, 30(1), 542-579. 
Burke, D.M., \& Shafto, M.A. (2004). Aging and language production. Current Directions in Psychological Science, 13(1), 21-24.

Clark-Cotton, M.R., Williams, R.K., Goral, M., \& Obler, L.K. (2007). Language and communication in aging. In J.E. Birren (Ed.), Encyclopedia of gerontology: Age, aging, and the aged (2nd ed., pp. 1-8). London: Elsevier.

Connor, L.T., Spiro, A., Obler, L.K., \& Albert, M.L. (2004). Change in object naming ability during adulthood. The Journals of Gerontology: Series B, 59(5), 203-209.

Deltour, J.J. (1993). Echelle de vocabulaire Mill Hill de J.C. Raven. Braine-le-Chateau, Belgium: Editions l'Application des Techniques Modernes.

Desrochers, A., \& Bergeron, M. (2000). Valeurs de fréquence subjective et d'imagerie pour un échantillon de 1,916 substantifs de la langue française. Revue canadienne de Psychologie Expérimentale, 54(4), 274-325.

Feyereisen, P. (1997). A meta-analytic procedure shows an agerelated decline in picture naming: Comments on Goulet, Ska, and Kahn (1994). Journal of Speech, Language, and Hearing Research, 40(6), 1328-1333.

Goral, M., Spiro, A.I., Albert, M.L., Obler, L.K., \& Connor, L.T. (2007). Change in lexical skills in adulthood: Not a uniform decline. Mental Lexicon, 2, 215-238.

Goulet, P., Ska, B., \& Kahn, H.J. (1994). Is there a decline in picture naming with advancing age? Journal of Speech and Hearing Research, 37(3), 629-644.

Hillis, A.E., Rapp, B., Romani, C., \& Caramazza, A. (1990). Selective impairment of semantics in lexical processing. Cognitive Neuropsychology, 7(3), 191-243.

Hough, M.S. (2007). Incidence of word finding deficits in normal aging. Folia Phoniatrica et Logopaedica, 59(1), 10-19.

Howard, D., \& Patterson, K. (1992). The pyramids and palm trees test. Bury St Edmunds, UK: The Thames Valley Test Company.

Lima, S.D., Hale, S., \& Myerson, J. (1991). How general is general slowing? Evidence from the lexical domain. Psychology and Aging, 6(3), 416-425.

MacKay, A., \& Burke, D.M. (1990). Cognition and aging: A theory of new learning and the use of old connections. In T. Hess (Ed.), Aging and cognition: Knowledge organization and utilization (pp. 213-263). Amsterdam, North Holland: Elsevier Science.
MacKay, A., Connor, L.T., Albert, M.L., \& Obler, L.K. (2002). Noun and verb retrieval in healthy aging. Journal of the International Neuropsychological Society, 8(1), 764-770.

Majerus, S., Lekeu, F., Van der Linden, M., \& Salmon, E. (2001). Deep dysphasia: Further evidence on the relationship between phonological short-term memory and language processing impairments. Cognitive Neuropsychology, 18(5), 385-410.

Mariën, P., Mampaey, E., Vervaet, A., Saerens, J., \& De Deyn, P.P. (1998). Normative data for the Boston Naming Test in native Dutch-speaking Belgian elderly. Brain and Language, 65(3), 447-467.

Morrison, C.M., Hirsh, K.W., \& Duggan, G.B. (2003). Age of acquisition, ageing and verb production: Normative and experimental data. The Quaterly Journal of Experimental Psychology, 56(A), 2-26.

Mortensen, L., Meyer, A.S., \& Humphreys, G.W. (2006). Agerelated effects on speech production: A review. Language and Cognitive Processes, 21(1/2/3), 238-290.

Myerson, J., Ferraro, F., Hale, S., \& Lima, S. (1992). General slowing in semantic priming and word recognition. Psychology and Aging, 7(2), 257-270.

Neumann, Y., Obler, L.K., Gomes, H., \& Shafer, V. (2009). Phonological vs sensory contributions to age effects in naming: An electrophysiological study. Aphasiology, 23(7-8), 1028-1039.

Nicholas, M., Connor, L.T., Obler, L.K., \& Albert, M.L. (1998). Aging, language, and language disorders. In M.T. Sarno (Ed.), Acquired aphasia (pp. 413-449). San Diego, CA: Academic Press.

Nicholas, M., Obler, L.K., Albert, M.L., \& Goodglass, H. (1985). Lexical retrieval in healthy aging. Cortex, 21(1), 595-606.

Salthouse, T.A. (1996). The processing-speed theory of adult age differences in cognition. Psychological Review, 103(3), 403-428.

Schmidt, R., Freidl, W., Fasekas, F., Reinhart, B., Grieshofer, P., Koch, M., ... Lechner, H. (1994). Mattis Dementia Rating Scale. Neurology, 44, 964-966.

Schyns, T., \& Poncelet, M. (2002). Cognitive Processing Speed Task. University of Liège, Belgium: Unpublished test.

Samson, D., Pillon, A., \& De Wilde, V. (1998). Impaired knowledge of visual and non-visual attributes in a patient with a semantic impairment for living entities: A case of a true category-specific deficit. Neurocase, 4(4-5), 273-290. 